\title{
Coverage Hole Problem Under Sensing Topology In Wireless Sensor Network
}

\author{
Nan Qu, Xiangjun Xiong \\ School of Science \\ East China University of Science and Technology \\ Shanghai, China \\ e-mail:xxj@ecust.edu.cn
}

\author{
Fang-Ming Shao* \\ School of Science \\ East China University of Science and Technology \\ Shanghai, China \\ e-mail: fmshao@ecust.edu.cn
}

\begin{abstract}
In wireless sensor networks, one of the fundamental issues is the coverage problem. This is, whether a given region can be sufficiently covered by sensing disks of sensors? In this paper, we discuss the coverage-hole problem by using a sensing topology. From the sensing topology, we figure out the relation between the graph $G n, n+i$ and coverage holes, and obtain that if $m<3 n / 2-(5-(-1) n) / 4$, there must exist a coverage hole for the closed graph Gn,m, which has a cut point but no cut edge. Finally, the simulation shows that the proposed approach is effective for some nodes randomly distributed in a region.
\end{abstract}

Keywords-wireless sensor network; coverage; sensing topology

\section{INTRODUCTION}

Wireless sensor network (WSN) consists of tiny sensor nodes, deployed in a region. Each sensor node has sensing and communication capabilities to gather information from the environment and to deliver data to the base station. The user tries to understand and analyze the information from the monitoring region. However, the analysis result may be affected by the coverage of disks of sensor nodes, such as some parts or holes of the monitoring region are not covered. Hence, it is very important to understand the relation between coverage hole and position of nodes and whether there exists coverage holes or not in the monitoring region.

In the wireless sensor network, sensors collect information from their surroundings, and the sensing data are then aggregated to give a complete picture of the region. Then we attempt to make sure whether sensing disks of sensors has completely covered the interest area. This issue has been tackled by various researchers in the sensor networks community. Lecture [1-4] analyzed the connectivity and sensing coverage of WSN. The research efforts focused on sensing coverage in WSN, and some researchers discussed the minimal active node-set that can completely cover a monitoring region. [5,6]discuss how to combine consideration of coverage and connectivity maintenance in a single activity scheduling, and then [7] enhances the work to support general wireless sensor networks and got another result: "the communication range is twice of the sensing range" is the sufficient condition and the tight lower bound to ensure that complete coverage preservation implies connectivity among active nodes if the original network topology (consisting of all the deployed nodes) is connected. To solve the optimal problem, [8] introduces the weighted sensing topology and some strategies, such as finding the 'basic' maximal independent node-set and estimating the coverage area of a node-set, are proposed to design a heuristic algorithm.

To better understand the topology and sensing area, this paper considers the coverage problem under a sensing topology. From the sensing topology, we figure out the relation between the graph $\mathrm{Gn}, \mathrm{n}+\mathrm{i}$ and coverage holes, and obtain that if $m<$, there must exist a coverage hole for the closed graph Gn,m, which has a cut point but no cut edge and if $m<2 n-3$, there must exist a coverage hole for the closed graph Gn,m with no cut point. Finally, the simulation shows that the proposed approach is effective for some nodes randomly distributed in a region.

The rest of this paper is organized as follows. Section 2 lists some notations and gives the definitions. In section 3, we figure out the relation between the graph $G n, n+i$ and coverage holes. Section 4 gives some experimental results. The final section concludes the research.

\section{SENSING TOPOLOGY AND MODEL}

\section{A. Notations}

$G(V, E) \quad$ Graph with node-set $V$ and link-set $E$

$E_{\mathrm{s}} \quad$ Set of S-links, where S-link $(u, v)_{\mathrm{s}}$ is defined if there exist a common area sensed by both two nodes $u$ and $v$

$G\left(V, E_{\mathrm{s}}\right) \quad$ Graph with node-set $V$ and link-set $E_{\mathrm{s}}$

$d(u, v) \quad$ The distance between node $u$ and node $v$

$R \quad$ Communication range of a sensor node

$S(v) \quad$ Sensing area of sensor node $u$

$r \quad$ Sensing radius of a sensor node

$N(u) \quad$ The neighbor of node $\mathrm{u}$

BCC Be Covered Completely

Assumptions:

1. There are no two nodes located at the exactly same position;

2. Each sensor can do 3600 observations;

3. All sensors have the same sensing range $r$ or have sensing disks with the same size.

\section{B. Coverage Model}

The wireless sensor network can be denoted as a graph $G(V, E)$. There exists a link $(u, v)$ if the distance of $u$ and $v$ satisfies $d(u, v) \leq R$, where $R$ denotes the communication 
range of sensor nodes. $E$ denotes the set of all links. We call $G(V, E)$ communication topology. Let $C$ be a region in which sensors are deployed. We assume each sensor $v_{\mathrm{i}}$ can do $360^{\circ}$ observations and has sensing disk centered at the sensor $v_{\mathrm{i}}$, denoted mathematically as $S\left(v_{\mathrm{i}}\right)$, where $r$ be the radius of $S\left(v_{\mathrm{i}}\right)$ and $=1,2, \ldots, n$.

Definition 1. Let $V$ be a node set $\left\{v_{1}, v_{2}, \ldots, v_{\mathrm{n}}\right\}$ and $S(V)$

$=\bigcup_{i=1}^{n} S\left(v_{i}\right)$. A region $C$ is called to be covered completely

(BCC) by node set $V$ if $C \subseteq S(V)$.

Whether a region is covered completely by a node set $V$ depends on the position of sensor nodes. Besides the communication topology, the different position and sensing disks of sensor nodes forms a new kind of topology in which the BCC problem can be well analysis.

\section{Sensing Topology}

In $G(V, E)$, there exists a new topology as to sensing function of sensor nodes. The sensing link exists if the two sensors' disk intersects with each other, called S-link ${ }^{[8]}$. In another word, there exists a S-link $(u, v)_{\mathrm{s}}$ if sensing disks of $u$ and $v$ satisfy

$S(u) \cap S(v) \neq \varnothing$,

where $S(u)$ and $S(v)$ are sensing disks of $u$ and $v$ respectively. Let $E_{\mathrm{s}}$ denote the set of all S-links. The socalled sensing topology is consisted of all nodes in $V$ and $S$ links. This is, $G\left(V, E_{\mathrm{s}}\right)$ is the sensing topology of $G(V, E)$. For example, Fig. 1(b) is the sensing topology of Fig.1(a). On the other hand, There exists a C-link $(u, v)_{\mathrm{c}}$ if the distance of $u$ and $v$ satisfies $d(u, v) \leq R$, where $R$ denotes the communication range of sensor nodes. $E_{c}$ denotes the set of all C-links, and $G\left(V, E_{c}\right)$ is called the communication graph, denoted as $G\left(V, E_{c}\right)$, such as Fig.1(c) is the communication topology of Fig.1 $(a)$.

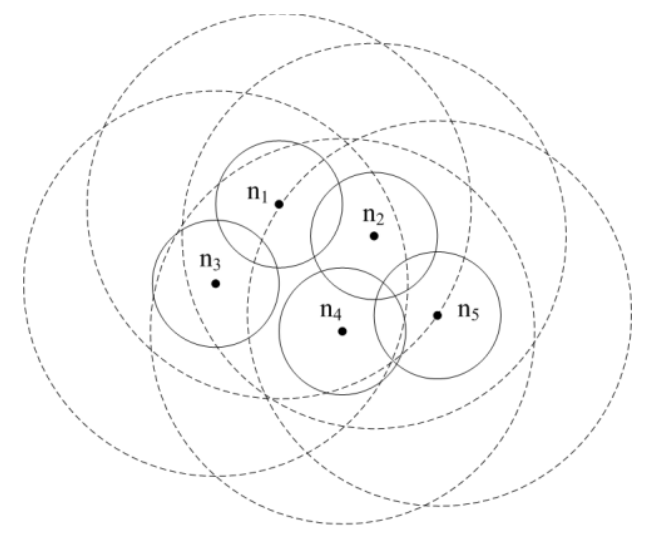

(a)

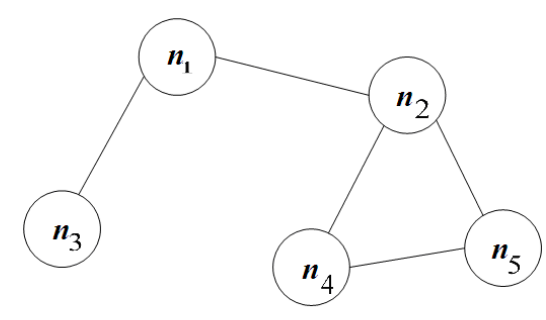

(b)

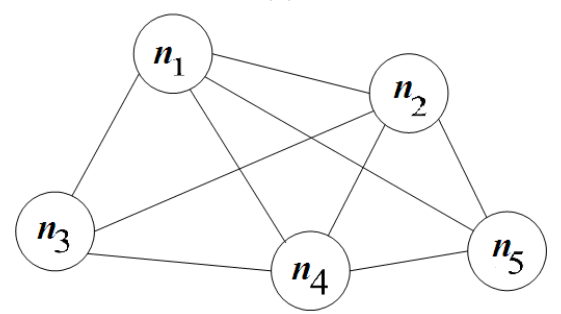

(c)

Figure 1. The sensing topology and communication topology of sensor nodes

Note: the real circle in Fig. 1 is sensing disk, and the dashed circle stands for communication circle.

\section{Coverage Holes}

Definition 2. A $G\left(V, E_{\mathrm{s}}\right)$ in sensing topology is called the closed graph if it doesn't contain any one-degree node.

BCC problem can be converted to whether the sensor node can cover their own area, which means, the monitor region is defined as an area that forms closely by most sensor nodes. In this case, we focus on the closed graph.

For example, in Fig.1(b), node 3 is one-degree node, there must be not covered completely if we count on the monitor region with node 3 . Hence it is meaningful if we consider whether the subgraph with 2,4,5 and their edges in Fig.1(b) is covered completely or not.

Definition 3. In the sensing topology, the closed graph $\mathrm{G}$ has a coverage hole if the area $C$ is not covered completely, where $C$ is a solid map drawn by border nodes of $\mathrm{G}$.

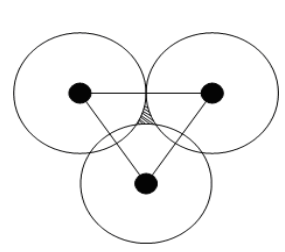

(a)

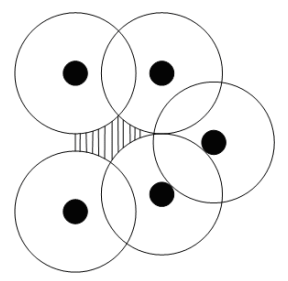

(b)
Figure 2. Disclosed network and closed network

When a network is closed, the region $C$ may still not be covered, such as the shadow illustrated in Fig. 2(a). The uncovered area (the shadow in Fig.2 (a)) is called coverage 
hole. However it does not mean that there exist coverage holes if the region cannot be covered completely. For example, the shadow in Fig. 2(b) is not coverage hole, because the graph is disclosed obviously.

\section{NeTwORK TOPOlOGY AND COVERAGE Hole}

Lemma 1. Let node $u$ and $v$ be any pair of nodes in $V$ satisfying $d(u, v) \leq 2 r$, and segment $m n$ is the vertical bisector of edge $u v$. Then there is not any node $y$ satisfying (i) $d(u, y) \leq 2 r$, (ii) $d(v, y)>2 r$, (iii) $m n$ interacts with $u y$.

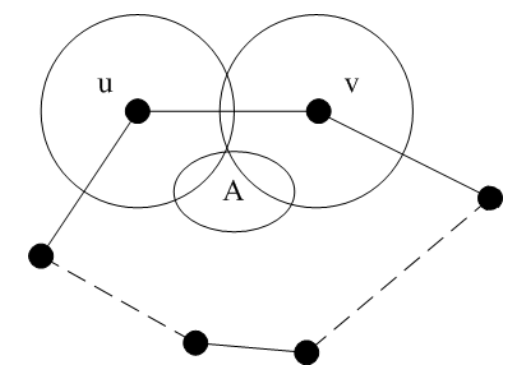

Figure 3. A graph with node $\mathrm{u}, \mathrm{v}$ and $\mathrm{y}$, and segment $\mathrm{mn}$ is a vertical bisector of edge uv

Lemma 2. Let node $u$ and $v$ be any pair of nodes in $V$ satisfying $d(u, v) \leq 2 r$, and if there is not a node $k$ in $V$ satisfying (i) $d(u, k) \leq 2 r$, (ii) $d(v, k) \leq 2 r$. Then there must exist coverage holes in $G\left(V, E_{s}\right)$.

Lemma 3. In closed graph $G\left(V, E_{\mathrm{s}}\right)$, if there is a region which can not be covered by a triangle, there must exist coverage holes.

Lemma 4. There must exist a coverage hole for the closed graph $G_{\mathrm{n}, \mathrm{n}}$, if $n \geq 4$.

In the following, three cases can be considered: a graph with a cut edge; a graph which has a cut point but no cut edge; a graph without cut point.

Theorem 1. For the closed graph $G_{\mathrm{n}, \mathrm{m}}$, which has a cut point but no cut edge, if $m<\frac{3}{2} n-\frac{5-(-1)^{n}}{4}$, there must exist a coverage hole.

Proof: According to lemma 3, a region in graph must be covered by a triangle, otherwise there must exist coverage holes. For the given graph $G_{\mathrm{n}, \mathrm{m}}$, we consider how many edges we need at least to make all the region in graph $G_{\mathrm{n}, \mathrm{m}}$ covered by triangles.

First, if there is a node in a triangle, the graph will increase at least three edges as illustrated in Fig.4(a). But if the node is out of a triangle, the graph will need only increase two edges or less, as illustrated in Fig.4(b). so we can conclude that there is no node in a triangle in the graph, if the graph has the least edges to make it covered.
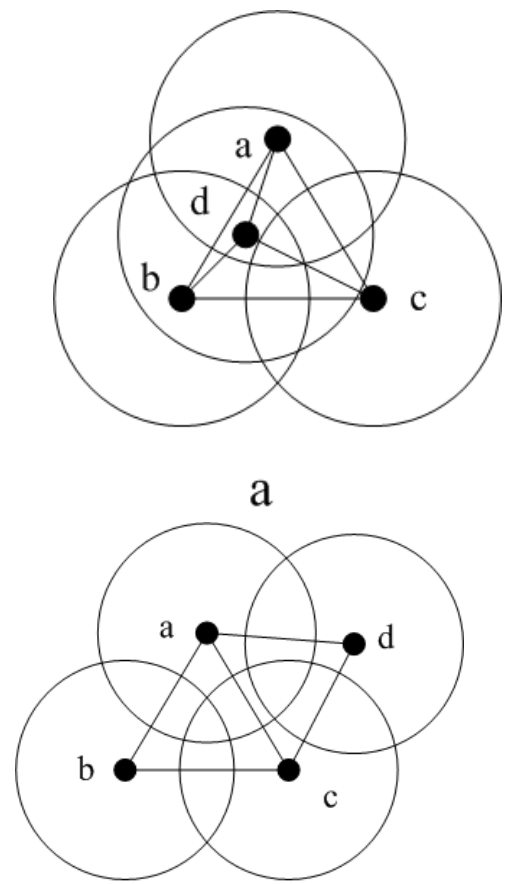

b

Figure 4. A node $d$ is in a triangle abc or out of a triangle $a b c$

Then according to the above and lemma 3, we know that the complete covered graph in Fig.5 with the least edges is showed as follows:

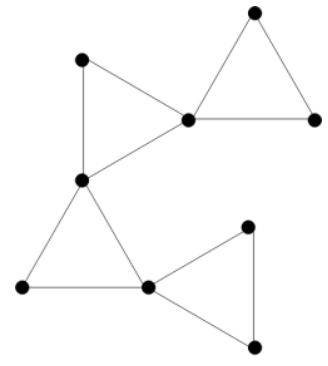

a

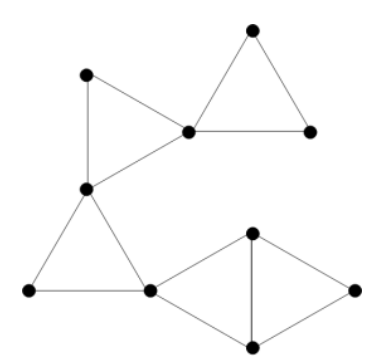

b
Figure 5. The complete covered graph with the least edges

Case 1. $n$ is an odd number, $m=\frac{3}{2} n-\frac{3}{2}, n \geq 3$

Case 2. $n$ is an even number, $m=\frac{3}{2} n-1, n \geq 4$

So the complete covered graph $G_{\mathrm{n}, \mathrm{m}}$ has the least edges, $m=\frac{3}{2} n-\frac{5-(-1)^{n}}{4}$. Hence while $m<\frac{3}{2} n-\frac{5-(-1)^{n}}{4}$, there must exist a coverage hole. $\square$. 


\section{EXPERIMENTAL RESULTS}

From Fig.6 (a), we can find the coverage holes from the sensing topology network and tackle it with the shadow. And we can judge the coverage holes directly by Theorem 1 .

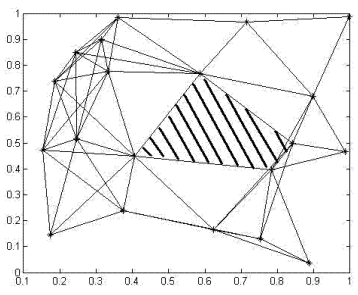

(a)

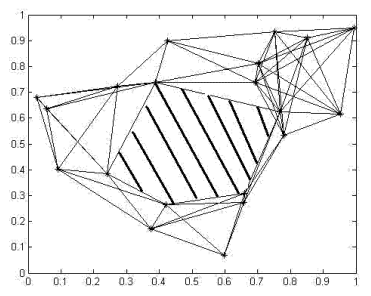

(b)
Figure 6. A network with 20 nodes uniform randomly placed in a 10-by10-m.

\section{CONCLUSION}

This paper considers the coverage hole in the defined sensing topology. In some cases, the coverage hole can be determined by counting the number of nodes and edges. The resulting researches prove the case that there exists a coverage hole. All research results can be used for the reliability and data analysis from the monitor area.

\section{ACKNOWLEDGMENT}

This work was supported by Shanghai Municipal Education Commission and Natural Science Foundation of China (61040040).

\section{REFERENCES}

[1] S. Meguerdichian, M. Potkonjak, Low power $0 / 1$ coverage and scheduling techniques in sensor networks, CLA Technical Reports 030001, 2003.

[2] S. Slijepcevic, M. Potkonjak, Power efficient organization of wireless sensor networks, in: Proc. IEEE Int'l Conf. on Communications ICC_01, Finland, 2001.

[3] D. Tian, N.D. Georganas, A coverage-preserving node scheduling scheme for large wireless sensor networks, in: Proc. ACM on Wireless Sensor Network and Application Workshop, 2002.

[4] T. Yan, T. He, J.A. Stankovic, Differentiated surveillance for sensor networks, in: Proc. 1st. ACM Conf. on Embedded Networked Sensor Systems, Los Angeles, 2003

[5] Xiaorui Wang, Guoliang Xing, Yuanfang Zhang, Chenyang Lu, Robert Pless, Christopher Gill, Intergrated Coverage and Connectivity Configuration in Wireless Sensor Networks, Proceedings of the First ACM Conference on Embedded Networked Sensor Systems, Los Angeles, November 2003

[6] Honghai Zhang, Jennifer C. Hou, Maintaining Sensing Coverage and Connectivity in Large Sensor Networks., Technical report UIUCDCS-R-2003-2351, June 2003

[7] Di Tian, Nicolas D. Georganas, Connectivity maintenance and coverage preservation in wireless sensor networks, Ad Hoc Networks vol.3, pp. 744-761, 2005

[8] Wei An, Fang-Ming Shao, Huajun Meng, The coverage-control optimization in sensor network subject to sensing area, Computers and Mathematics with Applications vol. 57 pp.529 - 539, 2009 